\title{
Evaluation of Transmissible Plasmodium falciparum infection in transfusion medicine
}

\author{
Abimbola Amoo ( $\nabla$ abimbolaamoo4@gmail.com ) \\ University College Hospital Ibadan https://orcid.org/0000-0002-6947-6707 \\ Hannah Dadaadegbola \\ University College Hospital Ibadan \\ John A. Olaniyi \\ University College Hospital Ibadan \\ Susan Gloria Omosa-Manyonyi \\ University of Nairobi College of Health Sciences \\ Humphrey Kariuki Njaanake \\ University of Nairobi College of Health Sciences
}

\section{Research}

Keywords: Transmissible, Malaria, Donor, Surveillance

Posted Date: July 29th, 2020

DOI: https://doi.org/10.21203/rs.3.rs-41859/v1

License: (c) (1) This work is licensed under a Creative Commons Attribution 4.0 International License. Read Full License 
TITLE: Evaluation of Transmissible Plasmodium falciparum infection in transfusion medicine

Author list: Abimbola Amoo (1,3) John Olaniyi (2) Hannah O. Dada-Adegbola (1) Kariuki Njaanake (3) Gloria Omosa-Manyonyi (3)

\title{
Affiliate Institutions:
}

1. Department of Medical Microbiology, University College Hospital Ibadan. Nigeria

2. Department of Haematology, University College Hospital Ibadan. Nigeria

3. Department of Medical Microbiology, University of Nairobi. Kenya

Corresponding author name and contact: Abimbola Amoo, abimbolaamoo4@gmail.com

\begin{abstract}
INTRODUCTION: In sub-Saharan Africa, Malaria could lead to about 770, 000 death alone this year if COVID-19 disrupts the key strategies, according to a newly available report on WHO modeling analysis. Transfusion medicine infections are mostly prevalent studies on bacteria and viruses with very little or no effort at investigating malaria parasite infection that can cause severe post-transfusion illness, especially in transfusion-dependent patients. OBJECTIVES: This study designed to bridge the gap by screening for Plasmodium falciparum infection and determine the effect of storage duration at $4^{\circ} \mathrm{C}$ on P. falciparum infection. METHODOLOGY: A Cross-sectional study on blood donors in Ibadan Oyo state, National blood transfusion service centre. Demographic data and clinical history were obtained using a pro-formal questionnaire. Donor's blood samples were P. falciparum positive using Giemsa stained microscopy. RESULTS: A total of 248 blood samples collected from donors from southwest Nigeria were tested for P. falciparum parasites. The overall prevalence of P. falciparum infection using Giemsa microscopy was $8.5 \%$. The prevalence in blood samples stored for 3days, 7 days, and 21 days were $8.1 \%, 7.3 \%$, and $5.7 \%$ respectively. There was a significant decline in the prevalence of P. falciparum, by microscopy, with an increased period of blood storage from day 0 to day 21 (Kendall's Tau; $\mathrm{p}<0.001$ ). CONCLUSION: The present study revealed that P. falciparum is prevalent among blood donors in the Ibadan, Nigeria. Blood storage for about 21 days can significantly reduce the risk of transfusion-transmitted P. falciparum malaria. This can be attributed to the lack of adequate accommodation and poor sanitary conditions in the area under study. National surveillance and public health education should stop the spread of parasitic infections in transfusion medicine.
\end{abstract}

KEYWORDS: Transmissible, Malaria, Donor, Surveillance 


\section{Introduction}

World Health Organization estimates high mortality if all key malaria control strategies are disrupted, and access to some of those interventions such as diagnostic testing are reduced by $75 \%$ as a result unprecedented level of collaboration in the fight against COVID-19 (WHO modeling analysis, 2020). Current transfusion blood screening services in the sub-Saharan Africa have focused mainly on viruses, such as HIV, Hepatitis B and C, and bacteria such as Treponema pallidum (Momoh et al. 2007). However, there is the need to consider other important pathogens such as Plasmodium spp, which have been implicated in transfusion medicine (Murphy, Evan \& Vermeulen 2013). Most of the developing tropical countries such as Nigeria have epidemiologic characteristics different from those in developed countries in addition.

They are characterized by the occurrence of endemic parasitic infections and diseases that are absent or rare in developed countries in addition to being plagued by the inadequate capacity to diagnose and manage the diseases (World Health Organization, 2008). The WHO recommends quality-assured screening of all donated blood for transfusion-transmissible pathogenssuch as HIV, hepatitis B, hepatitis C, Treponema pallidum (syphilis) and Trypanosoma cruzi, and Plasmodium spp (malaria) in some countries (Pondei, Lawani, \& Ndiok, 2012). In spite of this, blood screening for parasites is not routinely carried out in many parasite-endemic areas such as Nigeria (Oladeinde et al. 2014). The concern about HIV, hepatitis infection and transfusion transmission of COVID -19 has overshadowed the fact that other diseases, particularly parasites like Plasmodium spp. can be spread by transfusion and cause severe infection especially in immunocompromised patients (Attah E. B, 2000).

Blood donation is a highly regulated, safe process but screening for malarial parasites is not stipulated in the current National Blood Transfusion Guidelines. This is because transmission of parasitic microorganisms through blood transfusion is generally not regarded as a serious problem in an adult whose level of immunity is thought to be sufficiently effective in combating transfusion-transmitted parasitic infection in an endemic region (Federal Ministry of Health, 1991). Even during the stay at home COVID -19 pandemic, people still need critical health services. There would be continue roadtraffic injuries, pregnancy-related haemorrhage, cytotoxic chemotherapy, sickle cell anaemia, aplastic anaemia and thalassemia. Hence, the need for blood is constant, and regular blood transfusion which may be from different donors increase the possibility of transmission of blood-borne parasitic diseases (Attah E. B, 2000).

\section{Literature review}

Human malaria is a mosquito-borne disease caused by intraerythrocytic protozoa of the genus Plasmodium namely; P. falciparum, P. vivax, P. ovale, P. malariae and P. knowlesi. Plasmodium spp. require two hosts to complete their life cycle. The sexual cycle occurs in the vector female Anopheles mosquito and the asexual cycle occurs in human in two phases involving red blood cells (erythrocytic phase) and the hepatocytes (pre-erythrocytic phase). The incubation period of 83 mosquito-transmitted malarial infection is about 1 - 2 weeks but that of transfusion-transmitted malarial 84 infection takes about $2-4$ days. This is partly because transfusion-transmitted parasites by-pass the extra erythrocytic cycle (Karimi, Mardani \& Zadsar 2016).

The risk of acquiring transfusion-transmitted malaria is about $1-50$ cases per million donor units) in endemic regions including Nigeria. The factors influencing the prevalence of transfusion-transmitted malaria are seasonal variations with higher rates observed during the rainy season, criteria for blood 90 donor selection and the blood donor screening methods (Pondei et al. 2012). The majority of transfusion-transmitted malaria infections in Nigeria are caused by P. falciparum. According to Pondei et al. (2012) many blood donors in the endemic areas are asymptomatic carriers with parasite densities below the detection threshold of the currently available methods of diagnosis. These individuals have partially protective immune status and are generally the source of TTM (Pondei et al. 2012). A parasite identified in a thick blood film is equivalent to about 10,000 parasites in a $450-\mathrm{mL}$ unit of blood 
(Owusu-Ofori et al. 2013). This size of inoculum from transfused blood may pose a greater threat when compared to a bite from an infected mosquito, which has as few as 15 parasites (Owusu-Ofori et al. 2013). Malarial parasites can survive in blood cell for about 21 days outside the host (Owusu-Ofori et al. 2010). In Nigeria, screening for malarial parasite is not stipulated in the current National Blood Transfusion Guidelines (Faruk, Ogunrinde \& Mamman 2017).

This is because transmission of malaria through blood transfusion is generally not regarded as a serious problem in an adult whose levels of immunity is thought to be effective in combating transfusion transmitted malaria in an endemic region (National Blood Service 2006). However, the high number of blood transfusion-demanding health conditions such as in road traffic accidents, pregnancy-related haemorrhage, and severe immunosuppression enhance the possibility of the transmission of bloodborne diseases (Ewunife et al. 2011). Microscopic examination using Giemsa stained thick and thin blood films for malaria parasite sensitivity decrease with low parasite densities found in the blood of infected asymptomatic carriers. It is therefore, not sufficiently sensitive for blood bank service screening (Karimi, Mardani \& Zadsar 2016). Other methods of diagnosis include immunoassays such as indirect hemagglutination, Immunofluorescent assay, Radioimmunoassay, Enzyme latex immunosorbent assay, and antigen-based rapid diagnostic test, as well as molecular amplification methods such as using agar-gel diffusion and immunoassay to detect P. falciparum antigen and DNA. (Abdel-wahab et al. 2012).

\section{Methodology}

\section{Nature of the study}

This study was a cross-sectional study among 247 non-remunerated voluntary blood donors in National blood transfusion service centre Ibadan, Oyo State Nigeria, The study was carried out between the period June 2019 to February 2020. Nigeria. A random sampling technique was used to select all eligible and fit participants for the study, in the blood bank, 247 consenting donors aged between 18 and 59 years were randomly recruited following their written informed consent. Those that have been treated for malaria parasite last one month were excluded from the study.

The participants whole blood samples were taken from the cord of the blood bags and a giemsa stained of each participants slide was examined for P. falciparum under light microscope. The examination for $P$. falciparum was done on day $0,3,7$ - and 21 post sample collection.

\section{Laboratory investigation for P. falciparum by Microscopy}

Thick blood smears were prepared from each fresh donor blood sample and air dried, then stained with freshly prepared $10 \%$ Giemsa's stain solution for ten minutes. The stain was washed off gently and slides air-dried before they were examined under X 100 oil immersion microscope magnification. For positive slides, the number of parasites was counted against 200 leucocytes and quantification of parasite density estimated by assuming 8,000 leucocytes/ $\mu 1$ of blood, this was repeated on day 3 (f0), day 7 (f7) and day 21 (f21). Slides were reported negative when no P. falciparum detected in 100 fields of each thick smear. A sample with known high number of P. falciparum trophozoite was used as positive control.

\section{Data collection}

Data was collected using a self-administered semi-structured questionnaire. Data on sociodemographic characteristics were collected and Profile information about Plasmodium falciparum positivity such use of mosquito net, living riverine area, Blood group, and use of malaria prophylaxis. 


\section{Data management and statistical analysis}

Collected data were coded, cleaned and computed into Microsoft Excel spreadsheet and doublechecked for accuracy. Data on prevalence of P. falciparum were analyzed using STATA version 12 (Stata Corporation, College Station, Texas, USA). The categorical variables were summarized as proportions and frequencies. Test of association between/among qualitative variables was done using bivariate and multivariate analyses. The effect of storage duration on $\mathrm{p}$. falciparum density was compared using Kendall's Tau test, with the level of statistical significance set at a $\boldsymbol{p}$-value $<0.05$.

\section{Ethical considerations}

Ethical approval to conduct this study was obtained from Oyo State ethical review committee in Oyo state, Ibadan, Nigeria and UI/UCH Ethics Review Committee. This study was conducted under strict compliance with the guidelines of the Helsinki Declaration of 1964. Written and verbal informed consent was also obtained from all participant before they are enrolled into the study. The identity of all participants was treated with the utmost confidentiality.

\section{Results}

A total of 248 questionnaires were administered to healthy blood donors and the blood film microscopy of the respondents were analyzed for Plasmodium falciparum trophozoite positivity on initial day 0 , Nineteen of respondents were male, 13 were between age 29 and 39 year, 16 were Blood group $\mathrm{O}$ carriers. While about eight were sleeping under mosquito bed nets, and 21 were not on malaria prophylaxis during the sample collection. As shown in Table 1

\section{TABLE 1: BASIC PROFILE INFORMATION}

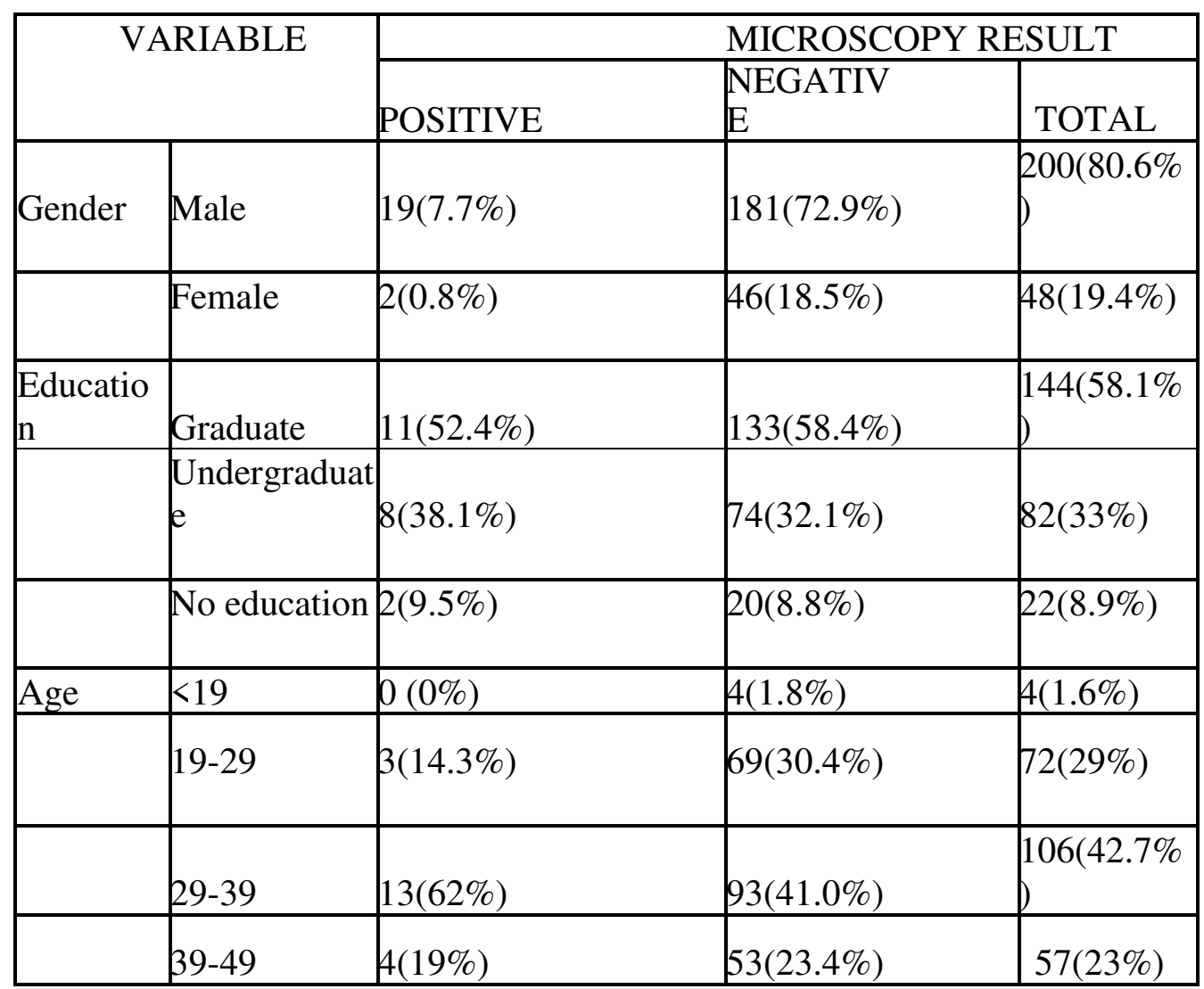




\begin{tabular}{|l|l|l|l|l|}
\hline & $49-59$ & $1(4.8 \%)$ & $8(3.5 \%)$ & $9(3.6 \%)$ \\
\hline $\begin{array}{l}\text { Mosquito bed } \\
\text { nets }\end{array}$ & Nets Use & $8(38.1 \%)$ & $114(50.1 \%)$ & $122(49.2 \%)$ \\
\hline & No net & $13(62 \%)$ & $113(49.8 \%)$ & $126(50.8 \%)$ \\
\hline $\begin{array}{l}\text { Malaria } \\
\text { Prophylaxis }\end{array}$ & Drug & $0(0 \%)$ & $4(1.8 \%)$ & $4(1.61 \%)$ \\
\hline & No drug & $21(100 \%)$ & $223(98.2 \%)$ & $244(98.4 \%)$ \\
\hline & & & & \\
\hline Blood groups & A & $4(19.1 \%)$ & $26(2.4 \%)$ & $2(11.45 \%)$ \\
\hline & B & $0(0 \%)$ & $25(11 \%)$ & $25(10.1 \%)$ \\
\hline & AB & $1(4.8 \%)$ & $5(2.2 \%)$ & $6(2.4 \%)$ \\
\hline & O & $16(76.1 \%)$ & $171(75.3 \%)$ & $187(75.3 \%)$ \\
\hline Zonal regions & Southwest & $16(76.2 \%)$ & $178(78.4 \%)$ & $194(78.2 \%)$ \\
\hline & Northwest & $1(4.8 \%)$ & $12(5.3 \%)$ & $13(5.2 \%)$ \\
\hline & North Central & $4(19.1 \%)$ & $28(12.3 \%)$ & $32(12.9 \%)$ \\
\hline & South south & $0(0 \%)$ & $9(3.9 \%)$ & $9(3.6 \%)$ \\
\hline & & & & \\
\hline
\end{tabular}

Figure 1: Prevalence of $P$. falciparum infection in follow-up stored blood samples by Microscopy

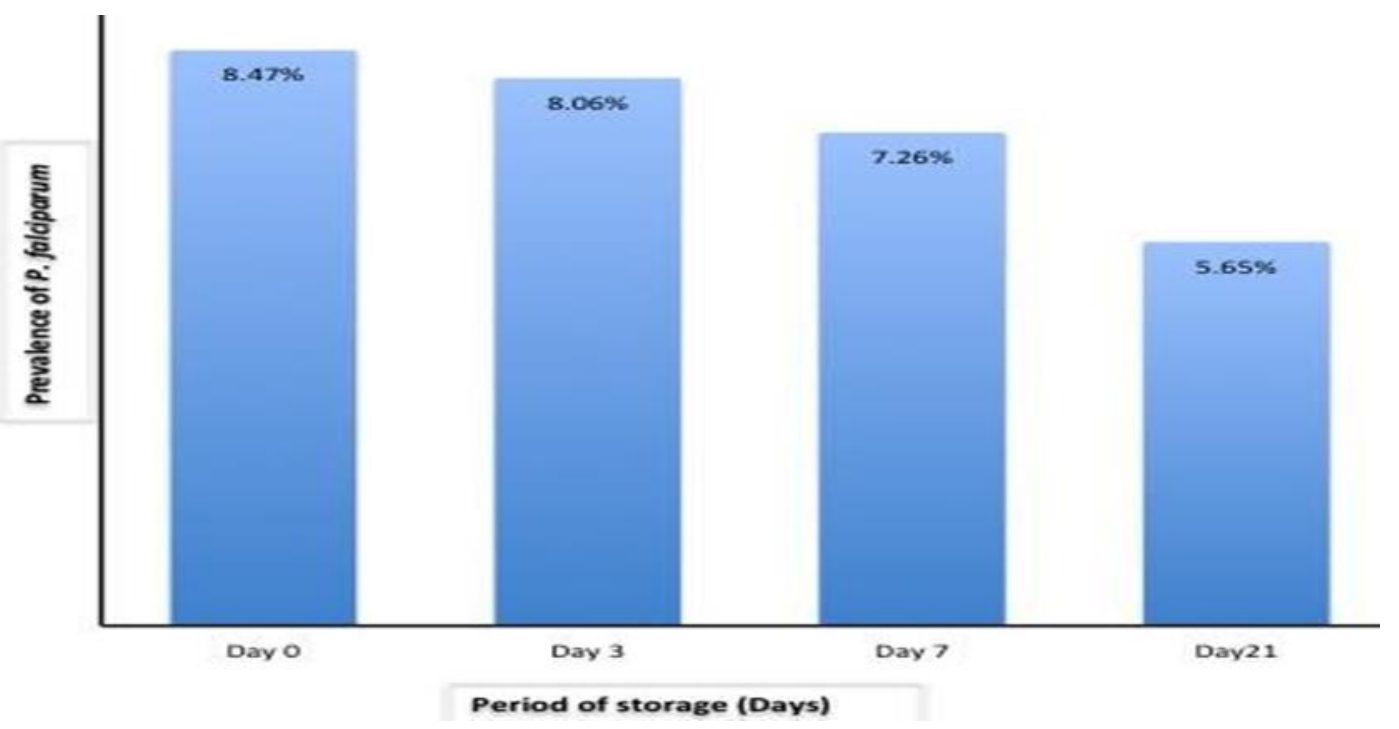


There was a significant difference in the prevalence of Plasmodium falciparum infection among the study participants microscopy $(8.5 \%)$ results on day $0(\mathrm{p}<0.001)$. There was a significant difference in prevalence of $P$. falciparum infection on day $3(8.1 \%)(p<0.001)$. The prevalence of $P$. falciparum infection on day 7 was $7.3 \%(p<0.001)$. The prevalence of $P$. falciparum infection on day 21, was $5.7 \%(\mathrm{p}<0.001)$ (Figure 1)

\section{The effect of storage duration on $P$. falciparum density}

During the baseline survey on day 0 and the three subsequent follow-ups at day 3, 7, and 21, the parasite density for the initially positive samples by microscopy ranged between 75 and 8679 parasite/ $\mu 1$ of blood. In the first follow-up, parasite density was between 37 and 7885 parasite/ $\mu 1$ of blood, the 3 days period of storage was associated with significantly lower parasite densities (Kendall's $\tau \mathrm{b}=0.9738, \mathrm{p}<0.001$ ).

In the second follow-up, after 7 days parasite density was between 36 and 3680 parasite/ $\mu 1$ of blood, the period of storage was associated with significantly lower densities of P. falciparum (Kendall's $\tau \mathrm{b}=0.9196, \mathrm{p}<0.001)$. the third follow-up $(14 / 248)$ was between 15 and 379 parasite/ $\mu 1$ of blood. The 21 days period of storage was associated with significantly lower densities of P. falciparum parasite (Kendall's $\tau b=0.8042, p<0.001$ ). Longer period of storage was associated with significantly lower P. falciparum densities.

\section{Discussion}

Blood samples were collected from blood donors and examined for P. falciparum trophozoites microscopy before storage at $4^{\circ} \mathrm{C}$. Part of the blood samples were stored at $4{ }^{\circ} \mathrm{C}$ and examined for $\mathrm{P}$. falciparum trophozoites after 3, 7, and 21 days using microscopy. The results showed a lower prevalence of $\mathrm{P}$. falciparum $(8.5 \%)$ on day 0 when compared to findings in similar study in Ibadan, Nigeria, amongst blood donors by (Okonkwo et, al. 2012). In Ghana, Owusu-Ofori et al (2013) reported a $13.7 \%$ among patient's blood donors. As observed by microscopy, the prevalence of $\mathrm{P}$. falciparum in the study population is low. This could be because the study was carried out, before the rainy season from January to April when malaria transmission is low (Chaponda et al., 2015).

There was a slight reduction in prevalence of $\mathrm{P}$. falciparum in the third day of storage at $4{ }^{\circ} \mathrm{C}$ compared to day 0 and steady decrease in P. falciparum prevalence to $7.2 \%$ and $5.4 \%$, on day 7 and 21 , respectively. There was also decrease in parasite density with increase period of storage indicating that storage in low temperatures has significant effect on P. falciparum (Chattopadhyay et al. 2015). Majority of blood donors were graduate, and there was a reduction in Plasmodium falciparum risk, this shows the level of awareness advocate among the elite blood donors. 


\section{Conclusion}

The overall prevalence of $\mathrm{P}$. falciparum during the study by microscopy on day 0 , first follow-up after 3 days, second follow-up after 7 days and third follow-up after 21 days of storage were $8.4 \%, 8.1 \%, 7.5 \%$ and $5.4 \%$ respectively. This implies that longer period of storage was associated with significant lower densities and prevalence. Malaria parasite reduce with duration of storage, therefore, the risk transmission through blood transfusion can be reduced, if the blood can be stored for a longer duration before transfusion.

\section{Acknowledgement}

The authors are indebted to the Staff of Hematology and blood transfusion department of University College hospital, Ibadan Nigeria for their support in collection of data and analysis of this project respectively.

\section{Conflict of interest}

All the authors declare no conflict of interest.

\section{References}

1. Abdel-Wahab, M. M, Ismail, K. A, El-Sayed, N M. 2012, 'Laboratory Diagnosis of Malaria Infection in Clinically Suspected Cases Using Microscopic Examination', OptiMAL Rapid Antigen Test and PCR. Pui, 5(1), pp. 59-66.

2. Agbolade, O. M, Akinmoladun, A, Abimbola, W. M, et al. 2013, 'Parasite among patients and blood donors in Ijebu-north, Southwest Nigeria', Asian Journal of Biological and Life Sciences. pp.185-189.

3. Attah, E.B. 2000,' Malaria and other Blood Parasites. In Human Pathology- A Complete Text for Africa (Attah EBS ed.) 1st.ed. Ibadan University Press, Ibadan. 203-210.

4. Chattopadhyay, R. N., Majam, V.F., \& Kumar, S. (2011). Survival of Plasmodium falciparum in human blood during refrigeration. Transfusion, 51 3, 630-5.

5. Cullen, K.A, Arguin, P. M 2014, 'Centers for Disease Control and Prevention (CDC). Malaria surveillance-United States, 2012. Morbidity and mortality weekly report. Surveillance summaries (Washington, D.C.: 2002), 63(12), pp.1-22. Available at: http://www.ncbi.nlm.nih.gov/pubmed/25474160.

6. Eunice, C. A, Ozumba, N. A, Eneanya, C. I. et al. 2011, 'Malaria infection among blood donors in Onitsha Urban, Southeast Nigeria. Sierra Leone Journal of Biomedical Research, 3(1), pp. 21-26.

7. Eze, U. A, \& Eze, N. M. 2015, 'Emerging Molecular Methods for the Diagnosis and Epidemiological Study of Parasitic Infections. An ASCII Journal of Health, 2(4), pp.3243.

8. Faruk, J. A, Ogunrinde, G. O, Mamman, A. I. 2017, 'Observation of Blood DonorRecipient Malaria Parasitaemia Patterns in a Malaria Endemic Region. Journal of TropicalMedicine, pp. 5. Available at: https://doi.org/10.1155/2017/71492612017. 
9. Federal Ministry of Health 1991, 'Guidelines on Appropriate Use of Blood and Blood Products for Nigeria. The National Blood Transfusion Service, Federal Ministry of Health, Lagos 1991; 1-51.

10. Federal Ministry of Health 2005, 'Nigerian National blood policy revised. The National Blood Transfusion Service, Abuja.p.16.

11. Israel, G. D. 1992, 'Determining Sample Size 1. Program Evaluation and Organizational Development, IFAS, University of Florida. POD-5. pp.1-5.

12. Momoh, A. R, Okolo, P.O, Okogbo, F. O, Orugbe, P. O. et al. 2007, 'Prevalence of blood pathogens among transfused patients in Ekpoma, Nigeria. International Journal of Community Research; 2(4): 72-76. Available at: http://www.arpjournals.com

13. Murphy, E, Evan, B, Vermeulen, M. 2013, 'Blood Transfusion Safety in Africa: A Literature Review of Infectious Disease and Organizational Challenges. NIH Public Access. 26(2), pp.164-180.

14. Murray, J. K, Bennet, J.W 2009, 'Rapid Diagnosis of Malaria. Interdiscip Perspect Infect Dis, pp. 1-7. Available at: doi:10.1155/2009/415953

15. Nagwa Mostafa El-Sayed. 2015, 'Recent Updates in Transfusion Transmitted Parasitic Diseases. Aperito $\mathrm{J}$ Bacteriol Virol Parasitol, 2:1 Available at http://dx.doi.org/10.14437/AJBVP-2-110

16. Okonkwo, I. O, Adejuwon, O. A, Okerentugba, P. O et al. 2012, 'Circulating Plasmodium falciparum and HIV $1 / 2$ as Co-infections among Blood Donors in Ibadan, Southwestern Nigeria. Nature and Science. 10(9), pp.42-47. Available at: http://www.sciencepub.net/nature

17. Oladeinde, H, Omoregie, R, Osakue E. et al. 2014, 'Asymptomatic malaria among blood donors in Benin City Nigeria. Iranian Journal of Parasitology, 9(3), pp.415-422.

18. Owusu-Ofori, Alex K, Parry J, Bates I. 2010, 'Transmitted Malaria in Countries Where Malaria Is Endemic: A Review of the Literature from Sub-Saharan Africa. Clinical infectious diseases: an official publication of the Infectious Diseases Society of America, 51(10), pp.1192-1198. Available at: http://www.ncbi.nlm.nih.gov/pubmed/20929356.

19. Owusu-Ofori, Alex K, Parry J, Martha B, Christopher M. et al. 2013, 'Transfusiontransmitted malaria in Ghana. Clinical infectious disease: an official publication of the Infectious Diseases Society of America, 56(12), pp.1735-41. Available at: http://www.ncbi.nlm.nih.gov/pubmed/23463635. 
Figures

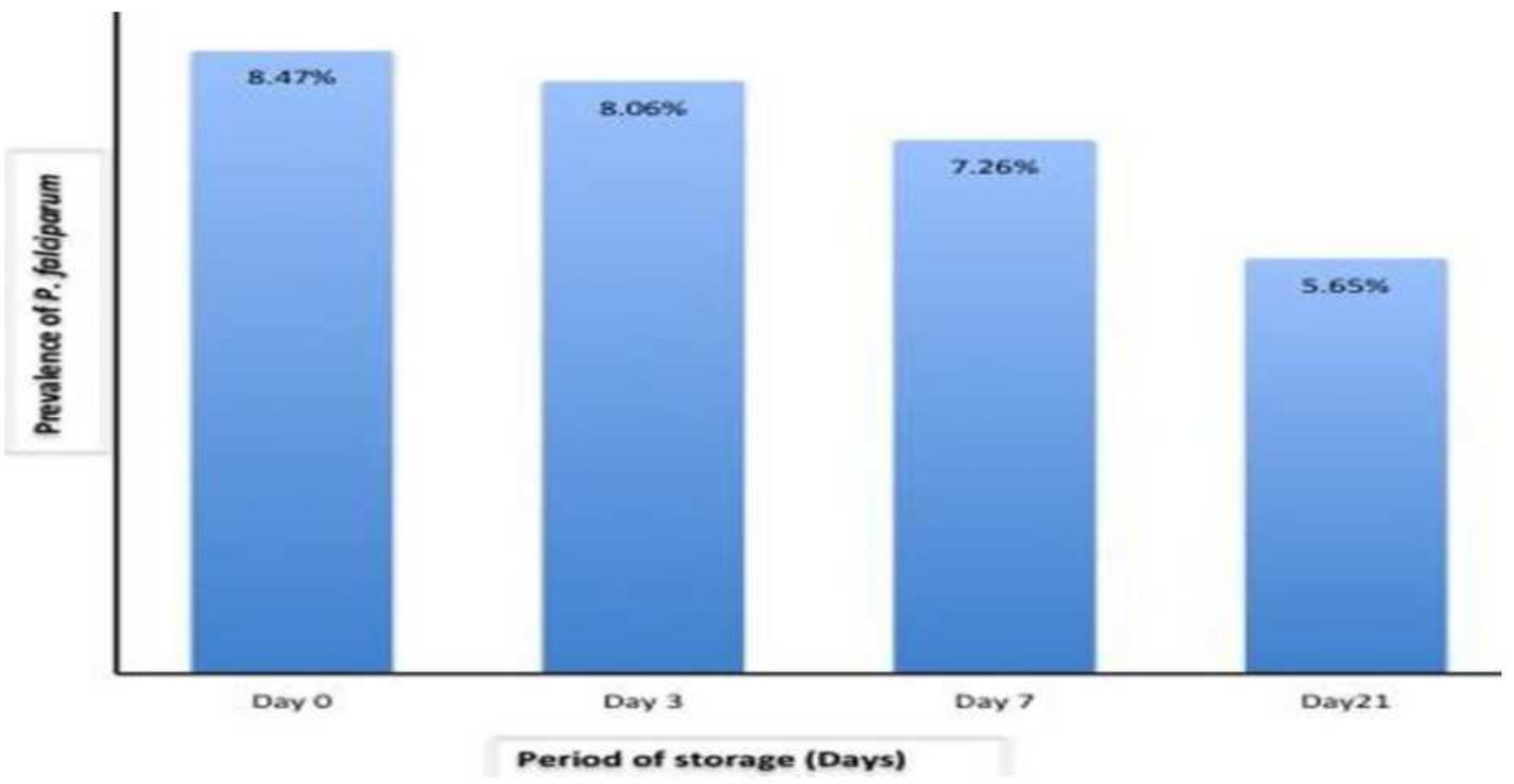

Figure 1

Prevalence of P. falciparum infection in follow-up stored blood samples by Microscopy 